\title{
Un modèle polyphonique en épistémologie sociale
} Croyances individuelles, pluralité des voix et consensus en matière scientifique39-58

\section{Alban Bouvier}

\section{(2) OpenEdition}

\section{Journals}

Édition électronique

URL : http://journals.openedition.org/ress/574

DOI : $10.4000 /$ ress. 574

ISSN : $1663-4446$

Éditeur

Librairie Droz

Édition imprimée

Date de publication : 1 août 2002

ISBN : 2-600-00806-3

ISSN : 0048-8046

\section{Référence électronique}

Alban Bouvier, "Un modèle polyphonique en épistémologie sociale », Revue européenne des sciences

sociales [En ligne], XL-124 | 2002, mis en ligne le 01 décembre 2009, consulté le 19 avril 2019. URL

http://journals.openedition.org/ress/574; DOI : 10.4000/ress.574 
Alban BOUVIER

\title{
UN MODÈLE POLYPHONIQUE EN ÉPISTÉMOLOGIE SOCIALE.
} CROYANCES INDIVIDUELLES, PLURALITÉ DES VOIX ET CONSENSUS EN MATIÈRE SCIENTIFIQUE

\author{
I. PRÉAMBULE: \\ ÉPISTÉMOLOGIE SOCIALE ET SOCIOLOGIE \\ DE LA CONNAISSANCE
}

\section{L'objet de la sociologie de la connaissance}

Le problème essentiel de la sociologie de la connaissance et auquel on conviendra aisément que se ramènent tous les autres est de savoir ce qui est social dans la connaissance, spécialement dans la connaissance scientifique, ou en quoi la connaissance, tout spécialement scientifique, est collective. Cela requiert, préalablement, de savoir ce qu'on entend par «social» et par «collectif» et on peut dire que la sociologie de la connaissance depuis Durkheim et Mannheim a donné des réponses nombreuses et variées à ces questions, sans pour autant, du reste, épuiser le sujet. En revanche, la sociologie de la connaissance s'est, en général, beaucoup moins posée la question de savoir ce qu'il faut entendre par «connaissance» ou par «connaissance scientifique» et s'est plutôt le plus souvent contentée de soutenir un continuisme plus ou moins tacite tendant à atténuer fortement ce qui distingue la connaissance scientifique de la connaissance commune ou de la simple croyance ${ }^{1}$.

\section{L'objet de l'épistémologie individuelle (individual epistemology)}

C'est au contraire la spécificité de l'épistémologie ou, plus généralement de la théorie de la connaissance - mais le terme anglais epistemology recouvre les deux et je prendrai ici épistémologie en ce sens large - que de se préoccuper de définir rigoureusement ce qu'on entend par connaissance scientifique et ce qui distingue celle-ci de la simple croyance. Depuis l'Antiquité, on se pose, par exemple, la question de savoir si la connaissance est simplement la croyance vraie ou si cette croyance vraie, pour être connaissance, ne requiert pas d'être assortie de justifications. On peut tomber sur le vrai par hasard ou on peut répéter quelque chose de vrai sans être capable de dire pourquoi c'est vrai. Platon appelait cela opinion vraie pour la distinguer de la science et la théorie contemporaine de la connaissance retrouve souvent ces questions très élémentaires en les renouvelant par un certain nombre de raffinements hérités de la longue histoire des théories de la

\footnotetext{
Il y a, bien entendu, des exceptions notables, comme Boudon \& Clavelin (1994).
} 
vérité ${ }^{2}$. Il est arrivé fréquemment - et il arrive encore - que ce genre de questions soit résolu en se référant aux seules facultés du sujet individuel: sans le dire, l'épistémologie (ou la théorie de la connaissance) est en réalité individualiste. Un des exemples les plus souvent cités de cet individualisme épistémologique est évidemment Descartes, même si cet exemple n'est pas pur, l'ego cartésien devant recourir à la garantie divine pour s'assurer de la validité des évidences les plus primitives au-delà du moment même où elles lui apparaissent dans toute leur clarté. Il est possible aussi de diviser les difficultés et d'envisager séparément ce qui relève du sujet individuel - mais seulement en un premier temps. Dans ces limites, l'épistémologie individuelle (individual epistemology) est alors légitime (Goldman, 1986).

\section{L'objet de l'épistémologie sociale (social epistemology)}

La social epistemology, l'épistémologie sociale est un prolongement de cette interrogation épistémologique générale, mais elle se pose la question plus spécifique de savoir ce qui entre de social dans la connaissance et si une épistémologie purement individuelle est seulement pensable ${ }^{3}$. Elle surenchérira, par exemple, à propos de la question de la justification d'une croyance vraie ou bien corroborée en ajoutant une autre question, que je formulerai sommairement ainsi: qu'est-ce qu'une justification qui ne convainc que son auteur? Ne faut-il pas qu'elle convainque aussi les autres et qu'elle finisse par être partagée? Comprise largement cette discipline englobe même en droit des théories de la connaissance comme celle d'Habermas qui font de la communication un a priori de la connaissance possible plus primitif que les individus ou les sujets eux-mêmes ${ }^{4}$. Ainsi donc sociologie de la connaissance et épistémologie sociale se rencontrent dans leurs questionnements, quoique avec chacune leurs accentuations propres.

\section{L'épistémologie sociale naturalisée}

Toutefois, si les champs respectifs sont assez marqués, les deux disciplines se distinguent surtout idéal-typiquement. Ainsi, certains des travaux qui se réclament de l'épistémologie sociale et qui le font à bon droit parce que leur perspective reste centrée sur la question de la validité de la connaissance ou, plus précisément, de sa validation au travers des interactions entre chercheurs, offrent-ils en fait surtout un éclairage sur ce qu'il faut entendre par «social» et «collectif» en science. Et c'est à ce qui entre de sociologie plus ou moins explicite dans les tra-

2 Cf. notamment, fondateur de ce renouvellement, l'article célèbre de Gettier (1963). Pour une typologie des théories contemporaines de la vérité du point de vue qui nous intéresse ici, cf. Goldman (1999), chap. 2.

3 Pour une présentation d'ensemble de l'épistémologie sociale à partir des limites de l'individualisme épistémologique, présenté lui-même dans ses différentes variantes, voir, par exemple, Schmitt (1999).

4 Certains auteurs cherchent désormais à reprendre explicitement les idées d'Habermas (voire de Foucault et d'autres auteurs de la philosophie continentale) dans le cadre de l'épistémologie sociale et de la nouvelle tradition de recherche que celle-ci constitue, caractérisée par un style beaucoup plus analytique que celui habituel dans la philosophie continentale. Voir, par exemple, Alcoff (1996). 
vaux d'épistémologie sociale que je m'intéresserai ici. La majeure partie de l'épistémologie sociale se conçoit, en outre, à divers degrés, comme naturalisée. Ce qui veut dire qu'elle a assumé, avec plus ou moins de radicalité, le tournant qu'a constitué, en théorie de la connaissance, la critique par Quine (1977) des grandioses programmes fondationnalistes empiristes de Carnap et du premier Goodman (les programmes rationalistes transcendantaux de type kantien ou husserlien étant déjà considérés comme caduques). Si ces programmes sont impossibles à remplir, si on ne peut fonder la connaissance qu'illusoirement (même sur une base empiriste), alors mieux vaut encore la décrire ${ }^{5}$. La social epistemology anglo-saxonne, celle d'Alvin Goldman (1999, 2002) et de Steve Füller (1988), par exemple, se veut non seulement normative, comme l'était l'épistémologie classique, mais elle se veut aussi descriptive. La social epistemology contient donc une sociologie du processus de connaissance, une sociologie qu'on peut appeler cognitive. De même que l'épistémologie simplement individuelle dont elle est le complément contient elle-même une psychologie cognitive comme l'une de ses composantes, quand, comme dans certaines versions radicales, elle ne tend pas à s'y réduire ${ }^{6}$.

\section{La variété de l'interactionnisme social et du constructionnisme social}

Mon propos spécifique sera toutefois nettement plus circonscrit. L'un des courants les plus actifs de la sociologie de la connaissance contemporaine comme de l'épistémologie sociale est le courant dit constructionniste. On peut le considérer comme une simple variété d'interactionnisme, c'est-à-dire de perspective considérant que le social est le produit de l'interaction entre des individus, et montrant comment la connaissance scientifique est sociale ou collective en tant qu'elle est le produit de l'interaction entre chercheurs, entre chercheurs et techniciens, etc. Au niveau le plus élémentaire, cela veut seulement dire que la connaissance scientifique n'est pas ou pas d'abord, contrairement à ce que des représentations ultraindividualistes, largement entretenues par la mythologie du génie créateur et illustré par quelques figures légendaires, pourraient laisser penser, essentiellement le produit de l'activité de la recherche personnelle. Des versions moins banales et

Quine (1977) écrit ainsi: «Mieux vaut découvrir comment la science se développe et s'apprend en réalité, que d'inventer une structure fictive dans la même intention» (p. 92) et: «L'épistémologie, ou quelque chose de ressemblant s'est simplement conquis droit de cité à titre de chapitre de psychologie et donc de science naturelle» (p. 96). Dans la mesure où un tel programme cherche, par définition, les conditionnements naturels de la connaisance, on ne doit qu'à peine s'étonner qu'une branche - au demeurant très dynamique - de l'épistémologie sociale naturalisée se développe dans le courant féministe puisque la différence entre les sexes est inscrite dans la nature. Voir notamment Nelson (1990) - dont le titre, Who Knows : From Quine to a Feminist Empiricism, est éloquent - ainsi que le recueil d'Alcoff \& Potter (1993).

6 Voir, par exemple, sur ce parallélisme, Goldman (1986), p. 1-3 et Goldman (1999) p. VII-X, le premier de ces ouvrages étant consacré explicitement à l'individual epistemology et le second à la social epistemology. Quine est bien plus radical que Goldman: la dimension normative tend à être tout à fait éliminée. Pour une comparaison entre ces deux formes d'épistémologie naturalisée, cf Engel (1996), respectivement pp. 344-54 et pp. 366-78 et, sur la difficulté qu'il y a à assumer l'idée de norme dans une épistémologie naturalisée comme celle de Goldman, cf Jacob (1989), cité par Engel (1996). 
plus radicales - pas forcément très élaborées dans leurs argumentaires - du constructionnisme social vont jusqu'à soutenir que non seulement la connaissance mais la réalité elle-même est sociale, donnant naissance alors à une nouvelle forme d'idéalisme. Des versions plus modérées et nettement plus raffinées, comme celle de Searle (1998) posent la question de savoir si le social peut se construire à partir d'intentions individuelles ou s'il ne faut pas déjà disposer d'intentions collectives, notion dont il faudra évidemment, dans cette perspective, examiner le contenu de sens.

\section{Le modèle interactionniste dialogique et polyphonique de Mara Beller. Soi multiple (multiple self) et infra-individualisme méthodologique}

Je voudrais m'arrêter à une autre version modérée et également raffinée d'interactionnisme qui se développe, elle aussi, à l'écart de tout relativisme et qui montre notamment, quant à elle, comment la pensée apparemment la plus individuelle peut être profondément sociale. L'idée de base est de donner un sens spécial à l'idée d'interaction, de manière à ne pas s'arrêter au simple constat de réseaux de relations ou à la simple forme quasiment géométrique des interactions, comme dans l'analyse dite de réseaux (utilisant la théorie des graphes) - ce qui serait rester à l'extérieur du contenu des théories - mais de montrer comment, par cette forme d'interaction qu'est la conversation scientifique, la controverse ou, plus généralement le dialogue, le contenu même des pensées apparemment les plus personnelles est social. La pointe la plus acérée de cette idée se trouve dans le concept de polyphonie qui permet de mettre en évidence comment, même en l'absence de dialogue effectif, c'est rarement d'une seule voix que le scientifique s'exprime. A son corps défendant souvent, son discours porte la marque du discours des autres, donc de leur «voix ». C'est Bakhtine qui a développé de la façon la plus fine cette idée, avant qu'elle soit notoirement reprise et retravaillée par un linguiste français, Oswald Ducrot dont, faute de place, je ne dirai rien de plus ici. Mais la même idée bakhtinienne a été plus récemment reprise en histoire et philosophie de la physique sur l'exemple de l'émergence et de la diffusion de l'interprétation de l'Ecole de Copenhague en mécanique quantique par Mara Beller (1999). Je ne suis pas physicien et je retiendrai seulement, en le dégageant du cas singulier, le modèle que Beller construit au fur et à mesure et qui est de portée beaucoup plus générale. Ce modèle mérite l'intérêt notamment parce que, alors que le dialogisme s'inscrit naturellement dans un cadre strictement individualiste, au sens où ce sont des individus qui dialoguent entre eux, la notion de polyphonie conduit à descendre à un niveau inférieur - infra-individualiste, donc - et à assumer une notion comme celle de pluralité du soi, voire de multiplicité du soi (multiple self), que certains partisans de la théorie du choix rationnel, comme James Coleman (1990, chap. 19) ou Jon Elster (1986), trouvent nécessaire d'introduire, forçant donc les limites de l'individualisme méthodologique ${ }^{7}$. La présentation - et la stylisation - de ce modèle polyphonique constituera la première partie de l'analyse qui suit.

Je discute d'autres variétés d'infra-individualisme - avec d'autres préoccupations - dans Bouvier 2002 . 


\section{Un modèle symétrique: la théorie du sujet pluriel de Margaret Gilbert appliquée à la notion de consensus scientifique. Un supra-individualisme (ou un micro-holisme) méthodologique}

Ce type de démarche me semble présenter d'autant plus d'intérêt que, d'un autre côté et de façon générale, l'individualisme méthodologique, sous une forme interactionniste ou non, est critiqué actuellement de divers côtés, alors même qu'il correspond à un niveau spécifique d'analyse. Il est critiqué par certaines théories naturalistes, par exemple de type darwinien, qui considèrent que le niveau d'analyse requis, de façon générale, dans l'explication des phénomènes sociaux est bien en deçà du niveau de la conscience des sujets, fussent-ils intimement «multiples », et qu'il faut chercher des invariants de la connaissance dans des catégories naturelles innées. Mais l'individualisme méthodologique est critiqué également par un retour du holisme durkheimien ou maussien sous diverses formes, qui va parfois chercher des renforts chez Wittgenstein. On peut, du reste, trouver des traces de holisme chez Kuhn lui-même, dans l'idée d'un consensus s'imposant aux chercheurs dans le cadre de la science dite normale.

Mais je voudrais m'arrêter plutôt, en un second temps, à un débat beaucoup plus proche des confins de l'individualisme méthodologique parce qu'il permettra d'affiner et de compléter les analyses précédentes en montrant quels prolongements elles appellent. Il s'agira, en effet, d'introduire à une forme de supra-individualisme ou de micro-holisme qui a le mérite de montrer comment des entités supra-individuelles ou micro-collectives peuvent être, en quelque sorte, engendrées à partir d'individus - de même que, sur le versant infra-individualiste, on pourrait montrer comment l'individu est, en quelque sorte, «dérivable» d'entités infra-individuelles. Ce micro-holisme, qui se réclame parfois de Simmel (Gilbert, 1989) est illustré par la théorie du sujet pluriel de Margaret Gilbert (1989, 1996, 2000). Son exposé et sa mise en relation avec le modèle infra-individualiste antérieurement exposé constitueront le second moment de mon analyse. Le sens général de mon propos sera donc de rattacher le débat sur ce qui entre de social et de collectif dans la connaissance scientifique aux débats du même type en théorie sociologique générale.

\section{ARGUMENTATION, DIALOGISME ET POLYPHONIE}

\section{Un point de vue argumentativiste}

Le modèle polyphonique, qui donne son nom à cet article est, en fait, l'étage ultime d'un modèle qu'on peut présenter sur trois niveaux: argumentativiste, dialogique, polyphonique. L'un des représentants majeurs de l'épistémologie sociale, Alvin Goldman, énonce explicitement qu'un des chapitres de ce domaine doit être la théorie de l'argumentation, c'est-à-dire, sur le versant descriptif, l'analyse des processus effectifs par lesquels des arguments avancés pour justifier une théorie convainquent ou non et, sur le versant normatif, la mise en évidence des règles auxquelles une bonne argumentation doit obéir (Goldman, 
1999, ch. 5) ${ }^{8}$. Un tel programme est évidemment nettement plus rigoureux que celui, fort répandu, qui, par le repérage de quelques traits rhétoriques de la science, conclut à la valeur très relative de celle-ci. Mais le cadre que propose Goldman reste très formel et donc forcément assez vide. Pour l'enrichir, l'affiner et, de ce fait, le rendre plus adéquat à la réalité à analyser et à évaluer, il est nécessaire de se donner des objets plus concrets. Ainsi, en ce qui concerne l'analyse d'argumentations, il est clair que l'on peut aller nettement plus loin dans la description des procédures effectives. Un bon moyen est de se tourner vers des études d'histoire des sciences étudiant des cas singuliers lorsque ces études sont sensibles à la dimension sociale de la science tout en restant très fermes sur le maintien des normes épistémologiques. L'une d'entre elles, précisément, celle de Mara Beller déjà citée, adopte un point de vue qu'on pourrait dire argumentativiste en ce qu'elle propose - je cite - de «traiter la réponse adressée à un public comme l'unité épistémologique et sociale primitive de l'analyse des sciences » (Beller, 1999, p. 308). Ainsi, poursuit-elle, «la notion d'une pensée scientifique présuppose l'existence d'un interlocuteur auquel la pensée est adressée ou par les énoncés duquel la pensée a été déclenchée » (p. 308). C'est là étendre à l'analyse du discours scientifique, y compris d'une des sciences les plus sophistiquées qui soit, la mécanique quantique, une conception que Perelman, le rénovateur de la théorie de l'argumentation, croyait précisément caractéristique du seul discours non scientifique. Ainsi Mara Beller analyse-t-elle constamment les arguments forgés pour justifier la croyance en la vérité d'une théorie ou, plus modestement, en sa validité, en tant que ces arguments sont adressés à des publics variés, tantôt à un public de spécialistes plus ou moins large tantôt à des individualités singulières en raison de leur compétence ou de leur expertise remarquable dans un domaine donné.

\section{Procédures d'argumentation}

Ainsi vers le public de non-spécialistes, Niels Bohr multiplie-t-il métaphores et analogies susceptibles de faire saisir, selon lui, la portée des concepts, comparant, par exemple, le quantum d'action et le concept de vie, la nature ondulatoire de la matière et le flux de la pensée, l'unité de la personnalité et l'individualité des particules matérielles... (Beller, 1999, p. 247) ${ }^{9}$. Le principe de complémentarité onde-particule se trouve ainsi étendu à la psychologie et à l'histoire et même à la théorie de la culture (p. 245). Pauli lui-même, le plus proche collaborateur de Bohr au moment de l'élaboration de ce principe, considère que sa portée est très exagérée par Bohr (p. 244) ${ }^{10}$. Il n'y a pas beaucoup à réinterpréter Beller pour voir aussi

8 L'argumentation n'est que l'une des «pratiques» que l'épistémologie sociale doit étudier. Elle doit s'interroger également sur d'autres modes sociaux de transmissions d'information, soit plus simples, comme le témoignage (testimony), soit plus complexes comme la technologie de la communication. Cf. respectivement, les chapitres 4 et 6 de Goldman (1999). J'ai cherché à défendre un point de vue systématiquement argumentativiste en sciences sociales en me référant à un autre cadre que celui de l'épistémologie sociale dans Bouvier (1999).

9 Ces analogies seront, à leur tour, comme le montre Terré (1998, pp. 176-194), à la source d'une véritable mythologie contemporaine.

10 Manifestement, la question de la portée de ce principe est très controversée. En général les auteurs prennent beaucoup plus au sérieux que M. Beller le principe de complémentarité, distinguant soi- 
que ce qu'elle appelle la «rhétorique de l'inévitabilité » (p. 193), dirigée cette fois vers les spécialistes pourrait être redécrite comme fondée sur un paralogisme de «monde clos» pour reprendre les analyses de Herbert Simon concernant ce qui bride les processus de découverte: la procédure consiste alors à convaincre qu'il n'y a pas de solution quand il y en a une ou qu'il n'y en a qu'une - inévitable quand il y en a plusieurs (en l'occurrence la solution alternative de David Bohm (Beller, 1999, p. 193)) ${ }^{11}$.

\section{Dialogisme et découverte}

Mara Beller donne encore de la densité aux distinctions qu'énonçait déjà Alvin Goldman dans sa propre analyse des types d'argumentation: elle rapporte en effet implicitement la distinction entre monologique et dialogique à deux moments différents de l'histoire d'une découverte: le moment dialogique est celui de l'émergence d'une nouvelle théorie. Elle montre, par exemple, comment tel échange avec Pauli ou Schrödinger est capital pour Heisenberg et, de façon générale, elle suit dans le détail l'élaboration du principe d'incertitude à partir de dialogues entre Heisenberg et tel ou tel physicien toujours identifié (Beller, 1999, chap. 4). C'est très explicitement qu'elle prétend ici prendre le contre-pied d'études qui pourraient, selon elle, s'inspirer de Lakatos mais, plus encore probablement, quoi qu'elle ne le cite pas, de Laudan, et parler de dialogues entre communautés (Beller, 1999, p. 314). Contre eux, elle adopte un interactionnisme fondé sur un strict individualisme et parle exclusivement de dialogues entre individualités. Mais Beller s'oppose encore à cette forme de holisme subtil qui consisterait à penser que même s'il y a dialogue entre individualités celles-ci seraient en même temps, voire d'abord les représentants de communautés. Beller montre que, au moins dans certains cas, comme celui de la genèse de ce qu'on appelle l'Ecole de Copenhague, rien ne permet d'accréditer cette idée: les chercheurs n'ont pas de croyances fixées, qui pourraient être caractéristiques d'une communauté, ils oscillent constamment dans le moment de la genèse de leur théorie et ils ne sont engagés à l'égard d'aucun paradigme. On pourrait dire qu'il y a des relations entre eux mais qu'ils forment plus une société qu'une communauté, pour reprendre une distinction célèbre depuis Tönnies, ou qu'ils appartiennent à divers cercles sociaux, mais que ceux-ci n'ont pas une grande stabilité, en tout cas pas propre à exercer une réelle pression sur leur mode de pensée. Beller étend son modèle à d'autres exemples et montre ainsi que cette absence d'engagement à l'égard d'une position donnée dans le moment de l'émergence d'une théorie serait valable d'Einstein aussi pendant qu'il élabore la théorie de la relativité générale. Ainsi, contrairement à ce qu'on dit habituellement, Einstein n'était pas engagé aussi fermement à

gneusement, par exemple, différents degrés d'extension de la portée du principe (Bitbol, 1999). Ils peuvent aussi s'inspirer de lui pour fonder leur conception des rapports entre philosophie de l'esprit et mécanique quantique (Bitbol, 2000, pp. 265-266), ce qui peut laisser a priori nettement plus sceptique.

11 R. Boudon a cru pouvoir mettre en évidence un processus de ce type dans Les formes élémentaires de la vie religieuse de Durkheim (Boudon, 1990). Et il est certain que Durkheim donne, de façon générale, l'impression d'une inévitabilité de ses conclusions, de même qu'il minimise les divergences avec les membres de son école (par exemple avec Lévy-Bruhl). 
l'égard du réalisme, pas aussi fixement opposé au positivisme de l'école de Copenhague. On le verrait au contraire osciller entre réalisme et anti-réalisme (p. 281-282).

\section{Monologisme et consolidation. \\ L'exemple paradigmatique de la Conférence de Niels Bohr à Côme en 1927. Croyances privées et croyances affichées publiquement}

Dans le moment de la consolidation des théories, au contraire, il peut y avoir apparition d'un discours monologique ou d'apparence fortement monologique. Dans l'esprit de Mara Beller, cela veut dire fondamentalement qu'il n'y a plus trace de dialogue et donc de divergences, mais un discours exprimant apparemment une seule voix; l'existence de désaccords avec des membres extérieurs au groupe est a fortiori tue. L'exemple de l'Ecole de Copenhague est encore paradigmatique. Ainsi, dans la fameuse conférence prononcée au Congrès International de Côme, Niels Bohr a prononcé un texte censé, selon Mara Beller, représenter les croyances partagées du groupe ou, plus exactement, un texte qui prétendait - toujours selon Mara Beller - forger une représentation unifiée ${ }^{12}$. Une telle unification peut aussi se faire au travers de manuels, éliminant, par nature, toute trace de débats. Et c'est ce qui ce serait fait. Mais les manuels en question auraient été rédigés d'une manière telle qu'il était impossible, en les lisant, de savoir qu'il y avait une interprétation alternative de la mécanique quantique, celle notamment de Schrödinger, puisqu'il n'en était fait nulle mention. Au cours de cette période, les thèses prennent alors la forme d'un dogme (Beller, 1999, chap. 9). Beller prétend que cela correspond exactement à ce que Kuhn avait en vue lorsqu'il parlait de paradigme caractéristique de la science normale ${ }^{13}$. Or Beller montre sur l'exemple de la mécanique quantique, que l'interprétation rendue publique et sur laquelle tous les membres de l'Ecole de Copenhague prétendaient publiquement s'entendre continuait à être, en réalité, l'objet de discussions à l'intérieur même du groupe, comme en témoignent les diverses correspondances concernées ou l'analyse minutieuse de leurs publications respectives. On peut donc dire, en première approximation, qu'il y a une différence entre les croyances privées (en matière de mécanique quantique), croyances qui sont l'objet de communications, ellesmêmes privées, entre un très petit nombre de chercheurs et les croyances affichées

12 Mara Beller écrit: « le besoin d'offrir une explication unifiée, capable de contrer l'opposition, était l'une des raisons de l'obscurité de la conférence de Bohr à Côme, dans laquelle les différences entre les positions de Bohr et celle d'Heisenberg ont été atténuées» (p. 143). Beller parle d'un «front public uni» (p. 143), à cette occasion, contre l'interprétation rivale, soutenue notamment par Schrödinger. A l'appui de la thèse d'un tel front uni, Mara Beller note, d'une part, que la version de la conférence de Côme qui fut prononcée ne fut pas écrite par Bohr seul mais avec l'aide de Klein et Darwin (p. 142), d'autre part que, dans la version publiée dans Nature, Bohr atténua ses désaccords avec Heisenberg, accentuant en même temps ce qui le rapprochait de Pauli (avec qui il était en dialogue au moment de la réécriture de son article), enfin élimina, à l'inverse, les divers éloges de Schrödinger que la version prononcée contenait (p. 142). Plus loin, elle signifie que la volonté de former un front uni était largement partagée: «[...] Bohr, Heisenberg, Pauli et Born serrèrent les rangs au Congrès de Côme et au colloque Solvay à Bruxelles en 1927 » (p. 216). 
publiquement ${ }^{14}$ devant non seulement des non experts mais aussi ce qu'on appelle la «communauté» scientifique élargie mais qu'il faudrait plutôt appeler plus prudemment la «société scientifique ${ }^{15}$. Ces croyances-là, publiquement affichées, on pourra les appeler - en première approximation et sans paraître, au moins à ce stade de notre analyse, forcer les usages linguistiques - des croyances collectives. Mais on voit déjà que ce n'est sûrement pas dans le même sens du mot «croire». Pour affiner ici l'analyse, j'aurais besoin des instruments conceptuels proposés par M. Gilbert et des discussions auxquelles ils ont donné lieu.

\section{Polyphonie (I). Le cas d'Heisenberg dans l'article de 1927 consacré au principe d'incertitude. Voix consonantes et voix cachées. Pluralité des voix mais croyance individuelle une}

Mais ce n'est pas encore là ce qu'il y a de plus intéressant dans l'étude de cas de Mara Beller. Celle-ci distingue, en effet, au moins implicitement, beaucoup mieux que Bakhtine, le modèle polyphonique du modèle dialogique. La polyphonie, si je stylise encore un peu plus la distinction de Mara Beller, c'est la présence dans un discours monologique de plusieurs voix. Le discours monologique, c'està-dire celui qui n'est pas pris dans un dialogue explicite - comme c'est le cas au contraire dans une conversation, dans un débat de colloque ou dans une correspondance, peut ainsi garder la trace du discours de l'autre ${ }^{16}$. Cela est vrai, par exemple, lorsque le discours prend en compte les objections des autres en les formulant pour y répondre. La référence à des acteurs réels peut prendre une forme explicite par le recours, banal, à la mention du nom des auteurs des objections ou à la citation même de leurs propos. Mais ces renvois et citations peuvent avoir une fonction de mise en évidence non seulement de divergences mais aussi de convergences: il y a certes plusieurs voix, mais elles disent alors toutes la même chose; elles ne sont plus dissonnantes mais consonantes.

Beller ajoute que dans les cas considérés, par exemple celui de Heisenberg écrivant l'article fondateur sur l'incertitude (Heisenberg, 1927), on est loin de voir représentée équitablement la société de ce qu'on pourrait appeler les «co-énon-

14 On pourra dire évidemment que la différence entre privé et public est relative (une lettre envoyée est moins «privée» qu'un journal personnel), ce qui est exact. Dans le cas présent, le critère de démarcation existe et il est simple: le texte en question a-t-il été ou non publié? Ce qui a été privé peut évidemment devenir ultérieurement public (publication de la correspondance, voire d'un journal intime) et il importe donc de distinguer, dans l'analyse, les différentes étapes chronologiques.

15 C. Chevalley, lors de la communication (à Lausanne) d'où est issu cet article a contesté qu'on puisse parler d'interprétation publique unifiée avant les années 50. Mais il semble qu'elle veuille dire: une interprétation publique qui corresponde aussi aux croyances privées de ceux qui la soutiennent. Or ce n'est évidemment pas le cas en 1927 selon Mara Beller non plus. Il ne semble donc pas y avoir de désaccord entre C. Chevalley et M. Beller sur ce point. L'important pour la suite de mon propos est, de toutes façons, qu'il y ait bien eu, à un moment donné, une interprétation unifiée publique (que cela soit dès 1927 ou seulement après 1950), coexistant avec des croyances privées dissonantes entre elles et avec la représentation publique affichée. C'est en ce sens faible mais sociologiquement très intéressant qu'il y aurait «une interprétation de Copenhague». Si la datation de Beller est exacte, Beller fait apparaître une différence de nature sociologique et psychologique entre l'interprétation de Copenhague en 1927 et celle des années 50 (cf infra).

16 C'est aussi la définition de l'argumentation monologique que propose Goldman (1999), p. 131. 
ciateurs » de l'article car toutes les voix qui ont participé au dialogue effectif ne se retrouvent pas dans l'article en question. Certaines sont donc cachées. Les auteurs moins renommés, quoique ayant joué leur part, parfois de façon décisive dans l'émergence d'une nouvelle idée, n'ont pas été reconnus, soit effet de l'oubli involontaire d'Heisenberg, soit effet d'une certaine arrogance ou condescendance ${ }^{17}$.

Qu'en tirer pour notre propos? On peut observer ici l'expression publique d'une pensée individuelle ou l'affirmation publique d'une croyance personnelle offerte à la discussion publique, plus large que la discussion avec les seuls proches, mais qui n'est pas collective au sens où nous nous avons employé ce mot un peu plus haut puisque personne n'est tenu de dire ou de laisser entendre qu'il la partage et donc de s'engager sur elle comme c'était le cas de la conférence de Côme censée (dans l'interprétation de Beller, au moins) représenter un point de vue commun. La différence importante est dans l'idée d'engagement et je reviendrai plus longuement sur cette notion dans la seconde partie de cet article. Mais d'un autre côté, c'est bien le résultat en quelque sorte agrégé d'interactions entre individus: en ce sens plus faible, elle est bien un résultat collectif. Pour éviter des ambiguïtés on pourrait plutôt dire que cette croyance est sociale mais qu'elle n'est pas collective.

Du moins l'agrégat de croyances privées ayant concouru à forger la pensée créatrice d'un chercheur - Heisenberg, par exemple - s'est-il trouvé unifié par le penseur: une voix domine toutes les autres. Il y a donc franchissement d'un seuil: il y a bien une croyance individuelle rendue publique qui a une unité et qui est irréductible à la simple somme ou à la simple résultante des autres croyances individuelles. On peut dire que c'est une propriété émergente spécifique - ou, comme on dirait en philosophie de l'esprit, pour éviter la surcharge de sens de la notion d'émergence: une propriété «survenante»-quoique la croyance soit sociale (dans son origine), c' est bien une croyance pleinement individuelle (dans son résultat).

\section{Polyphonie (II). A nouveau le cas de la conférence de Niels Bohr à Côme en 1927. Voix dissonantes. Une croyance individuelle simplement «agrégée» proposée comme croyance collective?}

Mais les analyses d'exemples de Beller montrent que ce n'est pas toujours le cas et, en prolongeant celles-ci, on peut montrer que certains paradoxes intéressants s'ensuivent. Ainsi Beller montre-t-elle sur l'exemple de la conférence prononcée par Niels Bohr au Congrès de Côme, conférence célèbre pour son obscurité et dont l'obscurité même n'a pas été pour rien, dit-on, dans le culte dont Bohr a été progressivement entouré, que cette obscurité s'explique bien si on suppose qu'il y a différentes voix non seulement implicites mais dissonantes alors que, dans le cas de Heisenberg, les voix étaient rendues consonantes par la domination de la voix propre d'Heisenberg unifiant toutes ces voix ${ }^{18}$. Ce n'est pas, du reste,

17 Du coup, les historiographies qui ne se préoccupent pas de rétablir la genèse dialogique des œuvres ont enterré elles-mêmes des noms comme ceux de Campbell, Sentfleben, Duane, Zernike, Ising (Beller, 1999, p. 96).

18 Cette description est légèrement idéalisée. Mara Beller montre, en effet, que la polyphonie implicite et dissonante peut être observée - quoique à un degré nettement moindre - dans l'article 
que Bohr veuille ici spécialement cacher ses sources, c'est qu'il a semble-t-il du mal à unifier sa pensée. La difficulté à comprendre la conférence de Bohr ne serait, en effet, pas du même type que celle que des générations de physiciens ont eu à comprendre la théorie de la relativité générale d'Einstein. Dans ce dernier cas, on sait, d'après les témoignages des spécialistes du domaine que ce qui est difficile, c'est, d'une part, la présence de mathématiques abstruses, comme le calcul des tenseurs, nécessitant un entraînement spécial et une agilité d'esprit assez peu répandue (Eisenstaedt, 2002, p. 202-203), d'autre part l'accoutumance à des concepts contre-intuitifs que le chercheur a toujours tendance à repenser, quelle que soit sa bonne volonté, à partir des concepts newtoniens (Eisenstaedt, 2002, p. 210-214). Ici, au contraire, les mathématiques, pour ainsi dire absentes, sont loin de faire obstacle. Et si les principes que Niels Bohr introduit sont difficiles à comprendre, c'est qu'ils impliquent un certain nombre de contradictions, à moins que, comme le montre Beller, on ne leur fasse correspondre des voix implicites diverses et dissonantes ${ }^{19}$. On a donc ici une croyance individuelle qui est sociale dans son origine mais qui ne réussit pas s'unifier. Paradoxalement, dans ce cas-ci, différent de celui d'Heisenberg dans son article sur l'incertitude, la croyance n'est pas pleinement individualisée - ou, interprétation plus généreuse: elle peine au moins à se faire reconnaître comme pleinement individualisée. Elle reste en quelque manière - ou au moins, elle donne l'impression de rester jusqu'à un certain point - un agrégat de pensées d'origine différente: il n'y a pas une pensée

même d'Heisenberg sur l'incertitude (Beller, 1999, Chap. 5, notamment p. 105). Elle montre, en particulier, qu'il y a essentiellement deux voix, l'une et l'autre exprimant la pensée d'Heisenberg, mais l'une d'elle représentant «sa propre voix passée» (p. 115). Il est évident, de toutes façons, que s'il subsiste en quelque manière une pluralité qui n'est pas dépassée il est plus difficile de penser l'idée d'émergence d'un niveau spécifique. Mais ce n'est pas le lieu d'en discuter ici.

19 C. Chevalley (1998a) ne semble pas, en fait, soutenir une position très différente. Elle écrit ainsi que c'est une caractéristique de Niels Bohr que de réécrire inlassablement et jusqu'au dernier moment ses articles, jusqu'à l'obsession. D'où de grandes différences, en général, entre les textes allemands originaires et leurs versions anglaises publiées. Elle ajoute même: «Le cas du manuscrit de Côme est évidemment le plus spectaculaire» et rapporte un jugement au premier abord paradoxal porté par les partisans de Bohr quant à l'obscurité fameuse de cette conférence: mieux Bohr comprendrait une question, plus ses articles seraient obscurs... La raison en serait la suivante: «d'un brouillon à l'autre, Bohr voit de mieux en mieux où gît la difficulté et sa solution». C. Chevalley commente cette étrange explication: «Dès lors [Bohr] s'efforce d'indiquer toutes les raisons de cette solution, tous les arguments qui pourraient conduire sur d'autres pistes, toutes les restrictions à faire, etc » (p. 581). Mais la clarification réelle qu'apporte $\mathrm{C}$. Chevalley consiste très exactement, me semble-t-il, à reconnaître que le texte est polyphonique au sens technique, c'està-dire linguistique, de ce terme: les arguments conduisant vers d'autres pistes n'expriment pas la propre voix de l'auteur mais celle d'autres chercheurs; de même les restrictions. La difficulté, dans des cas de ce genre, c'est que la voix même de l'auteur peut-être comme assourdie par celle des autres ou se confondre avec elle. Si les marqueurs linguistiques indiquant qui prend vraiment en charge les arguments sont trop ténus, le lecteur a du mal à discerner la voix même de l'auteur et donc à saisir ce qu'il soutient vraiment. L'enchevêtrement des voix nuit, du coup, à la perception de l'orientation générale de l'argumentation. Plus de voix sont introduites (donc, peut-on penser, plus de problèmes sont soulevés, et plus est approfondi le traitement du sujet), plus l'identification de la voix propre de l'auteur devient difficile. Je me suis intéressé à des problèmes de ce genre sur l'exemple d'un texte philosophique classique, les Méditations de Descartes, dans l'analyse duquel j'utilise justement le modèle polyphonique au sens de Bakhtine-Ducrot (Bouvier, 1995 , chap. 6 et 7, Bouvier, 1996). 
individuelle une émergeant - ou émergeant clairement - du dialogue intérieur. Pas de voix qui domine nettement les autres.

Mais ce n'est pas tout encore: dans la mesure où un public se trouve fasciné par cette pensée, y compris un public scientifique de non-spécialistes ou de demispécialistes, cette croyance va se trouver partagée par un cercle plus large. Ceux dont je parle ici ne sont pas du tout ceux dont la voix se trouve exprimée dans cette croyance - puisque, justement, Heisenberg, Pauli, Max Born ne peuvent pas vraiment se retrouver dans la conférence de Côme (c'est-à-dire y croire authentiquement). Ce qu'il faut quand même appeler - pour l'instant - «croyance », même si le concept a manifestement ici une extension trop large, est une croyance à la fois sociale (dans son origine), pas ou peu individualisée (dans son résultat ${ }^{20}$ et déjà collective par l'accord public, au moins tacite, qu'un groupe de penseurs, en l'occurrence des membres de l'Ecole de Copenhague, lui ont donné. Mais il n'est pas sûr que, sur les questions même dont traite la conférence, parmi les différents individus du public scientifique - autres que ceux appartenant au cercle étroit de l'Ecole de Copenhague - qui partagent désormais aussi cette croyance, chacun ait, cette fois, une croyance privée clairement distincte de la croyance collective. On perçoit que les non-experts qui adoptent cette croyance collective ne peuvent être dits «croire » à cette théorie au même sens que les experts - dont la croyance privée, au moins, est unifiée - croient, quant à eux, à la théorie qu'ils professent et qu'évidemment ils comprennent ${ }^{21}$. Comme certains anthropologues et historiens nourris d'anthropologie l'ont fait remarquer (Sperber, 1982, Veyne, 1983), on ne peut réellement croire à ce qu'on ne comprend pas. Ces dernières remarques appellent des analyses complémentaires sur la nature des croyances collectives et l'exposé des analyses que Margaret Gilbert réserve aux croyances collectives en contexte scientifique nous y aideront.

\section{DU «JE» COMME SOI MULTIPLE ET POLYPHONIQUE AU «NOUS» COMME SUJET PLURIEL}

\section{Du niveau infra-individuel (soi multiple) au niveau supra-individuel (sujet pluriel) en passant par le niveau individuel : processus d'émergence}

J'ai développé, dans le moment précédent de mon analyse un certain nombre de thèmes qu'un modèle argumentativiste en épistémologie sociale, plus précisément dialogique et polyphonique, met au premier plan, en me fondant sur l'analyse d'un cas historique menée de façon particulièrement exemplaire par Mara

$20 \mathrm{Ou}$ - pour toujours mentionner la restriction plus haut introduite - dont l'individuation est malaisément reconnaissable.

21 On pourrait donc, à la limite, soutenir - s'il est vrai que la conférence de Côme n'est pas pleinement unifiée autour d'une voix dominante - que cette conférence de Côme n'exprime pas même la croyance privée de Bohr. En voulant intégrer à sa conférence la voix des autres, en mélangeant sa propre voix à la leur, il aurait construit une sorte de «chimère » une pensée qui ne serait la pensée d'aucun individu particulier, pas même la sienne. M. Gilbert (1994) envisage explicitement des cas de ce genre, mais sans donner d'exemple: «Il est posssible qu'un groupe croie que 'p' même si la plupart des membres du groupe ne croient pas que 'p'. Il se pourrait même qu'il ne soit pas nécessaire qu'un seul membre croie que 'p'» (p. 100). 
Beller. Toutefois, les problèmes classiques que Mara Beller entend éclairer relèvent de l'histoire épistémologique ou de l'épistémologie historique - c'est Kuhn essentiellement qu'elle discute - avant même que de relever de l'épistémologie sociale ou de la sociologie de la connaissance. Certains concepts comme ceux de croyance collective ou encore d'engagement, que j'ai utilisés de façon simplement allusive jusqu'ici, méritent d'être davantage analysés sur les exemples mêmes qu'elle propose.

Or, dans l'éventail des théories disponibles à ce niveau, l'une semble tout indiquée, la théorie du sujet pluriel de Margaret Gilbert. Cette théorie relève de la philosophie générale des sciences sociales ou de la philosophie sociale en général. Elle concerne, par exemple, le thème de la coordination ou celui des obligations politiques tout autant que celui de la connaissance. L'épistémologie sociale n'y est donc qu'un chapitre parmi d'autres et, réciproquement, s'il est arrivé à M. Gilbert de publier dans tel ou tel volume collectif consacré à l'épistémologie sociale, elle ne fait pas partie des spécialistes du domaine les plus «visibles», comme Alvin Goldman, Steve Füller ou Frederik Schmidt, par exemple.

Si la théorie du sujet pluriel (plural subject, plural subjecthood) s'impose pour ainsi dire à notre attention, c'est pour deux raisons essentielles: d'une part parce qu'il s'agit d'une discussion subtile de l'individualisme méthodologique - qui analyse le sens de concepts comme ceux de «collectif» ou de «social» sur le fond d'une connaissance très fine, qui plus est, de leurs usages effectifs en sciences sociales, notamment chez Durkheim et chez Simmel - d'autre part et plus encore, parce que le concept de sujet pluriel est, pour ainsi dire, le symétrique du concept de soi multiple et donc de polyphonie et qu'on peut y observer, à un autre niveau, les mêmes processus d'émergence, aboutis ou inaboutis, d'une entité supérieure. Il s'agissait notamment, dans les analyses précédentes, de voir comment l'individuel (une pensée une) pouvait émerger de l'infra-individuel (la polyphonie héritée du dialogue); mais il s'agissait aussi déjà, quoique plus implicitement, de voir comment le supra-individuel (une croyance collective, une interprétation scientifique consensuelle) pouvait émerger de l'individuel. C'est ce second problème que je reprend ici. On peut espérer que les perspectives s'éclaireront mutuellement, l'analyse de cas précédente densifiant l'analyse conceptuelle que je vais présenter en lui donnant un contenu en quelque sorte plus épais, celle-ci clarifiant en retour l'analyse historique en en dégageant la trame logique.

\section{Sujet pluriel (le «nous» ${ }^{22}$ ) et engagement conjoint dans les actions les plus banales de la vie quotidienne}

La notion de sujet pluriel est élaborée par M. Gilbert pour rendre compte du type d'acteur collectif minimal, celui à partir duquel on ne peut plus parler simplement d'individus sans perte, c'est-à-dire sans manquer quelque chose de la spécificité du social. C'est même, à strictement parler, seulement à partir du moment où on doit introduire cette notion d'un micro-acteur collectif - il suffit

22 La notion de sujet pluriel pouvant malheureusement évoquer aussi l'idée que le «je» est multiple puisque le «je» est aussi un sujet et même le sujet par excellence, je précise ici qu'il s'agit de cette forme de sujet collectif que le «nous » sert souvent à désigner linguistiquement - sinon toujours puisqu'il peut aussi désigner de simples agrégats de circonstances. 
d'être deux, mais dans une relation particulière que Gilbert décrit - que, selon Gilbert, on peut parler de fait social.

Il y a sujet pluriel - pour définir sommairement la notion - lorsque deux individus ou plus se sentent conjointement engagés l'un par rapport à l'autre ou les uns par rapport aux autres à faire une action ensemble. Cet engagement n'a pas besoin d'être explicite, il a pu suffire de regards, de gestes, de grommellements mêmes, dans les cas d'expériences de la vie quotidienne, ajoute M. Gilbert. Dans ces cas-là, on ne peut dire simplement que tel individu fait une action et que tel autre la fait avec lui ni que cette action collective est simplement le résultat d'intentions individuelles convergentes. Il est plus exact de dire, pour les individus en question: «cette action est nôtre » plutôt que de dire: «cette action est mienne et elle est aussi la tienne». Gilbert dit que le terme linguistique adéquat est ici «nous »: « Nous avons fait cette action». Cette action est le résultat d'une entente, qui supposait que nous nous sentions engagés l'un à l'égard de l'autre, de sorte que si l'un voulait faire défection, l'autre aurait été fondé, en raison d'une sorte d'obligation morale, à faire des reproches au premier.

Le cas le plus simple est celui d'un sujet pluriel consitué de seulement deux personnes. M. Gilbert (1996, chap. 6) prend comme exemple paradigmatique celui d'une promenade que deux individus se trouvent avoir fait sans l'avoir forcément vraiment décidé au départ. Ils pouvaient fort bien s'être rencontrés par hasard sur la route et s'être mis à cheminer ensemble. Mais à partir du moment où, d'un commun quoique tacite accord, ils se sont mis à marcher à la même cadence, chacun a contracté par rapport à l'autre une sorte de micro-obligation: continuer à marcher ensemble sans ni presser le pas ni le ralentir. Chacun sent que, s'il veut changer d'allure, il devra s'excuser par une formule du style: «je m'excuse mais je suis pressé, je dois me dépêcher». Même dans des cas aussi rudimentaires, donc, une sorte d'engagement a été contracté de chacun avec l'autre: ils se sont conjointement engagés à marcher au même rythme.

On notera que la relation n'est pas nécessairement égalitaire: les promeneurs peuvent fort bien occuper des positions hiérarchisées (par exemple un professeur et un doctorant sur un campus universitaire ou deux professeurs de renom inégal). Il n'empêche qu'ils sont engagés l'un et l'autre l'un par rapport à l'autre et pas seulement l'inférieur par rapport au supérieur: si le supérieur doit soudain hâter le pas, on s'attend à ce que même lui esquisse, d'une façon ou d'une autre, une formule d'excuse ou un geste en tenant lieu. Cela n'exclut pas, du reste, que dans des cas de ce genre, comme de façon plus générale, du reste, les individus qui se trouvent conjointement engagés à l'égard des autres dans un groupe - fût-ce une simple dyade, comme aurait dit Simmel - peuvent s'être sentis plus ou moins librement engagés à contracter obligation ${ }^{23}$. L'important est que, bon gré mal gré, ils aient accepté de participer à cette action, fût-ce sous le mode minimal de l'avoir « laissée faire» ou de l'«avoir laissée se mettre en place». Mais à partir du moment où ils

23 M. Gilbert (1989, 1996, chap. 6) compare son analyse aux théories classiques du contrat social, celle de Rousseau comme celle de Hobbes. Mais il est clair que la notion d'obligation qu'elle décrit relève plus d'une analyse hobbésienne que rousseauiste car il est essentiel au pacte rousseauiste que les individus se sentent librement engagés à contracter; ils ne peuvent librement s'en remettre à quelqu'un qui décide pour eux. «La liberté ne se délègue point». Seule l'exécution des décisions peut être déléguée. Rousseau s’oppose explicitement à Hobbes sur ce point. 
ont accepté ils sont engagés et, dès lors, pèse sur eux une sorte de contrainte collective (même s'il n'y a que deux personnes) qui vient de l'engagement pris à l'égard de chacun et qui ne vient que de cet engagement conjoint. M. Gilbert (1994), en s'appuyant sur l'idée de contrat, montre finement comment la contrainte collective dont parle Durkheim pourrait n'être rien d'autre que l'engagement conjoint des individus les uns à l'égard des autres. Cette contrainte collective serait donc bien la résultante des actions des individus et, plus précisément même, des engagements ou micro-engagements qu'ils auraient eux-mêmes contractés.

\section{Sujet pluriel («nous») et engagement conjoint sur des idées dans la vie quotidienne et au sein d'une communauté scientifique}

La théorie du sujet pluriel ne rend pas compte seulement des actions mais aussi des idées collectives. M. Gilbert (1994, p. 16-7) prend ainsi l'exemple, très trivial encore en tant que tel, d'un groupe de professeurs d'Oxford discutant informellement des menus du collège. L'un exprime un mécontentement marqué sur le fait qu'il y a trop souvent de la viande au repas, un second surenchérit en disant qu'il y a trop souvent des pommes de terre. Un troisième fait un hochement de tête en signe d'acquiescement. Un autre semble vouloir protester mais se ravise, un cinquième proteste effectivement mais n'est pas entendu et n'insiste pas. Deux autres semblent indifférents. Enfin, celui qui semble avoir le plus d'autorité naturelle dans le groupe conclut: «Il est clair que nous sommes tous mécontents du menu. Il faut que nous allions nous plaindre ». Ceux qui n'avaient rien dit ne disent rien encore, soit qu'ils sont légèrement intimidés par la détermination de celui qui a pris la parole, soit qu'il n'y accordent pas vraiment d'importance. Mais, en ne protestant pas ou pas de manière perceptible quand le leader a dit «nous », ils se sont comme trouvés incorporés à cette entité collective désignée par le «nous». C'est comme s'ils avaient donné tacitement leur accord. Il pourra y avoir conflit ultérieurement entre les membres du groupe, certains ayant le sentiment d'avoir été légèrement embrigadés, mais néanmoins, ils se sont eux aussi, en quelque sorte, engagés par leur silence même. Gilbert conclut: ne dira-t-on pas, à bon droit, que ce que ce groupe pense, c'est que les menus du collège ne sont pas bons? Même si, individuellement, ils sont d'une opinion contraire, ils se sont mis dans la position d'accepter cette opinion collective comme leur et de malaisément pouvoir protester si on la leur impute.

Margaret Gilbert (1994) prétend, de cette façon, réinterpréter de façon adéquate l'idée durkheimienne - plus particulière que celle, générale, de contrainte collective - selon laquelle il y a des croyances collectives qui ne sont pas réductibles à des croyances individuelles et qui ne peuvent être dites avoir pour sujet des individus (mais qui vont néanmoins s'imposer à eux). Réinterpréter, du même coup, l'idée durkheimienne selon laquelle il y a des sujets collectifs - les sujets de ces croyances collectives - émergents par rapport aux sujets individuels: le sujet de l'opinion commune serait un sujet pluriel, qui ne serait pas formé de l'addition de sujets individuels mais qui constituerait, au contraire ceux-ci en une entité spécifique, un corps dont ils seraient les membres ${ }^{24}$.

24 Nous laissons de côté ici les questions d'ontologie. Mais il est évident que ces sujets pluriels ne peuvent être dits «exister» au sens où les sujets individuels existent puisque seuls ces derniers 
Margaret Gilbert (2000, chap. 3) franchit une étape supplémentaire qui nous intéresse directement lorsqu'elle utilise ce modèle pour rendre compte des croyances scientifiques et de l'engagement collectif dans un paradigme. Gilbert est toutefois alors essentiellement intéressée à montrer comment il est difficile pour quelqu'un de s'affranchir du consensus de la communauté scientifique à l'égard duquel il a jusque là contracté une sorte d'engagement conjoint à dire qu'il le partage. Le but de Margaret Gilbert est de chercher à montrer comment - ou plus exactement par qui - le changement scientifique, le changement de paradigme est possible. Elle montre ainsi que le changement pourra plus facilement arriver soit par les néophytes dans le domaine soit par les outsiders étrangers par définition au domaine, les uns et les autres n'ayant jamais été encore engagés par rapport aux autres.

\section{L'exemple de la Conférence de Bohr à Côme relu à travers la théorie du sujet pluriel}

Mais le modèle que M. Gilbert forge est beaucoup plus riche que les exemples qui précèdent ne le suggèrent. On voit notamment qu'il s'applique assez remarquablement au cas de l'interprétation de la mécanique quantique proposée par Niels Bohr au Congrès de Côme (dans l'interprétation de M. Beller). Et ce qu'on peut mettre en évidence sur cet exemple empirique plus que ne l'a fait M. Gilbert dans ses analyses abstraites, c'est la dualité éventuelle de croyances dans les mêmes individus. Dualité de croyances qui n'est pas vécue comme contradictoire puisque, dans l'esprit des chercheurs concernés (ou des professeurs engagés dans une protestation triviale dans l'exemple de Gilbert), il est clair qu'il y a une différence de niveau entre ce qui est cru et exprimé comme tel en privé et ce qui est affiché en public comme croyance collective - mais qui peut ne correspondre nullement à une croyance authentique.

Ainsi, si Heisenberg, Pauli, Max Born resserrent les rangs autour de Bohr au Congrès de Côme puis au Colloque Solvay à Bruxelles en 1927, s'ils affectent en quelque sorte publiquement de pleinement partager l'interprétation de Bohr - ou, du moins, acceptent de ne pas publiquement s'en démarquer du tout - leurs divergences avec Bohr comme entre eux sont évidentes à la lecture de leurs propres articles et de leur correspondance. On peut donc dire que ce qu'on appelle l'Ecole de Copenhague est un sujet pluriel dans le sens même que Gilbert donne à ce concept. Ce n'est pas une simple collection d'individus, pas un simple agrégat, pas une simple «société». On peut bien parler de «communauté» scientifique. L'existence d'un sujet pluriel n'implique évidemment pas que l'interprétation de (l'Ecole de) Copenhague corresponde à quelque croyance individuelle que ce soit (encore moins à une croyance unifiée) mais elle implique, en revanche, l'idée d'engagement conjoint à professer des croyances communes ou, au minimum, à

sont des sujets empriques (ayant un corps, un cerveau, etc). Pour d'autres raisons, les entités infraindividuelles que nous supposions plus haut n'existent pas non plus au sens où existent les sujets individuels empiriques. Dans ce dernier cas, plus simple que celui des sujets pluriels, ces entités sont seulement la «réflexion» - au sens optique du terme - dans un sujet d'autres individus existants - ou «l'écho» de leur voix. Nous ajouterons, à ce propos, que Sperber et Wilson (1989) ne sont pas loin de cette interprétation du modèle polyphonique, même s'ils n'en parlent pas explicitement, lorsqu'ils parlent de l'aspect «échoïque» de la communication verbale (pp. 356-364). 
ne pas s'en démarquer publiquement. Et, comme dans le cas de la promenade tacitement engagée, si on veut prendre publiquement de la distance, il sera attendu des autres membres du groupe qu'on se justifie.

\section{Polyphonie et sujet pluriel dans le cas des croyances scientifiques et des croyances mythologiques}

M. Gilbert n'a pas imaginé d'exemples aussi complexes que ceux que fournit l'histoire de la mécanique quantique analysée au travers du modèle polyphonique. Dans le cas de la conférence de Côme, ce qui est remarquable, en effet, sous ce nouveau rapport, ce n'est pas tant que la croyance collective puisse ne correspondre à aucune croyance individuelle, c'est que la croyance en question puisse être contradictoire. Le modèle de Beller permet d'en trouver la source dans la présence de voix dissonnantes, elles-mêmes induites par l'origine dialogique de la pensée. M. Gilbert n'avait pas imaginé d'exemples de la vie quotidienne de ce type mais il faudrait se représenter un porte-parole des professeurs aigris formulant une synthèse alambiquée des différentes opinions et, du coup, une synthèse en quelque manière confuse. Dans le cas de ce que Beller appelle «la rhétorique de la consolidation », la présence de voix diverses est oblitérée ou, en tout cas, une voix domine clairement toutes les autres. Il pourrrait s'agir, dans l'exemple de Gilbert, d'un porte-parole faisant encore état d'opinions diverses mais hiérarchisant, cette fois, clairement celles-ci.

Le premier cas est, de loin, le plus intéressant, sociologiquement et anthropologiquement parlant car il est évident que si un savant peut avoir du mal à donner une unité par écrit à une interprétation scientifique, des mythes issus d'une multitude de reformulations au travers de traditions différentes seront a fortiori dissonants. Depuis longtemps les exégètes de la Bible, par exemple, ont formulé - en utilisant d'autres mots, mais c'est la même idée - l'hypothèse d'une profonde polyphonie implicite ${ }^{25}$. Mais, dire cela, c'est retrouver une observation faite par les anthropologues et notamment par Lévy-Bruhl, à savoir que les croyances collectives - que Lévy-Bruhl distinguait bien des croyances individuelles, ce qu'on oublie souvent - sont souvent contradictoires. La polyphonie de celles-ci pourrait en être l'une des causes.

\section{Croyances collectives ou acceptations collectives?}

On notera, avant de conclure, que la différence même de niveau - souvent répétée dans cet article - entre les croyances privées et les croyances publiques ou affichées publiquement crée une sorte de malaise que nous avons déjà signalé et que note M. Gilbert (1994) elle-même, puisqu'on utilise, dans les deux cas, le concept de «croyance» alors que les réalités subsumées sous le concept sont nettement distinctes. Mais on peut y remédier sans trop de difficultés. En s'inspirant de la distinction que fait l'épistémologue L.J. Cohen (1992) entre belief et acceptance (reprise par Stalnaker et Van Fraassen, que Gilbert (1994, n. 34, p. 25) cite

\footnotetext{
Pareto (1968), qui y voyait de façon un peu courte un simple expédient fallacieux (du type de l'hypothèse $a d$ hoc destinées en sciences à sauver une théorie de la contradiction), repérait ce genre d'interpétation dès les premiers Pères de l'Eglise.
} 
sans préciser ses références), on pourrait parler d' «acceptations » collectives plutôt que de croyances collectives, c'est-à-dire, d'idées que des individus acceptent et s'engagent même à soutenir sans forcément y croire réellement en eux-mêmes. Ainsi, en s'en tenant au niveau le plus élémentaire du débat, on peut dire, en choquant moins les usages linguistiques (en tout cas quand on utilise la forme verbale) et en étant phénoménologiquement plus exact, que Heisenberg, Pauli, Max Born, etc. acceptent collectivement - tel un seul corps (Gilbert écrit dans des cas similaires: «as a body ») - les idées énoncées par Bohr et qui constituent ce qu'on appelle «l'interprétation de l'Ecole de Copenhague». Leurs croyances stricto sensu, c'est-à-dire leurs croyances privées, en revanche sont différentes sur des points fondamentaux. Il n'est même pas certain que chacun ne se soit pas senti un peu forcé à s'engager contre son gré dans cette entreprise collective, comme tel ou tel professeur d'Oxford de la fable de M. Gilbert, mais la réalité de l'engagement contracté n'en est pas pour autant changée.

\section{CONCLUSION}

Je conclurai en revenant brièvement à l'une des idées initiales de cet article. Margaret Gilbert propose la notion d'un sujet collectif ayant en quelque sorte des représentations propres, notion qui fait de la théorie sociale qu'elle développe une forme de micro-holisme. Mais elle décrit le processus d'engendrement de ces entités collectives à partir des individus de sorte qu'on peut parcourir le chemin inverse et remonter de ces entités collectives, sujet ou croyances aux actions des individus qui en sont la cause. Ce holisme s'articule donc aisément avec un individualisme méthodologique. Sur l'autre versant, que je voulais ici spécialement mettre en relief, la notion de discours polyphonique telle que l'analyse Mara Beller suggère la notion d'un sujet multiple et éventuellement dissonant, conduisant, cette fois, à prolonger l'analyse individualiste vers le niveau infra-individuel. Si les analyses précédentes sont un tant soit peu convaincantes, la démonstration pourrait être ainsi faite que la sociologie de la connaissance scientifique et l'épistémologie sociale de la science pourraient constituer un domaine à partir duquel on pourrait poser de façon pertinente des problèmes généraux de théorie sociologique. L'un d'entre eux est précisément celui de l'élargissement de l'individualisme méthodologique ou de son dépassement - lui-même méthodique - vers d'autres niveaux d'analyse.

Institut des sciences humaines appliquées

Université de Paris IV

\section{BIBLIOGRAPHIE}

L. M. Alkoff \& E. Potter, Feminist Epistemologies, New York, Routledge, 1993.

L. M. Alkoff, Real Knowledge. New Versions of the Coherence Theory, Ithaca, Cornell University Press, 1996.

M. Beller, Quantum Dialogue. The making of a revolution, Chicago, The University of Chicago Press, 1999. 
M. Bitbol, Complémentarité, in D. Lecourt (dir.), Dictionnaire d'histoire et de philosophie des sciences, Paris, PUF, 1999, pp. 202-205.

- Physique et philosophie de l' esprit, Paris, Flammarion, 2000.

N. Bohr, The Quantum Postulate and the Recent Development of Atomic Theory, in Atti del Congresso Internazionla dei Fisici, 11-20 Settembre 1927, 1927, pp. 565-88 (cité in Beller, 1999).

R. Boudon, L'art de se persuader, Paris, Fayard, 1990.

R. Boudon et M. Clavelin (éds), Le relativisme est-il résistible?, Paris, PUF, 1994.

A. Bouvier, L'argumentation philosophique. Etude de sociologie cognitive, Paris, PUF, 1995.

- La cohérence de l'argumentation philosophique et les normes de la communication. Une perspective de sociologie cognitive, in F. Cossutta (éd.), Descartes et l'argumentation philosophique, Paris, PUF, 1996, pp. 43-84.

- Philosophie des sciences sociales. Un point de vue argumentativiste en sciences sociales, Paris, PUF, 1999.

- An Epistemological Plea for Methodological Individualism and Rational Choice Theory in Cognitive Rhetoric, in «Philosophy of the Social Sciences», vol. 32, 2002, n 1, pp. 51-70.

C. Chevalley, Mythe et philosophie. La construction de «Niels Bohr» dans la doxographie, Phûsis, 1998a, pp. 569-603.

- «Introduction»à Werner Heisenberg. Philosophie. Le Manuscrit de 1942, Paris, Le Seuil, 1998b.

L. J. Cohen, Belief and Acceptance, Oxford, Clarendon Press, 1992.

J. Coleman, Foundations of Social Theory, Cambridge, Harvard University Press, 1990.

J. Eisenstaedt, Einstein et la relativité générale. Les chemins de l' espace-temps, Paris, CNRS Editions, 2002.

J. Elster (ed.), The multiple self, Cambridge, Cambridge University Press, 1986.

P. Engel, Philosophie et psychologie, Paris, Gallimard, 1996.

S. Füller, Social Epistemology, Bloomington, Indiana University Press, 1998.

E. Gettier, Is Justified True Belief Knowledge?, in « Analysis », pp. 121-123, reed in E. Sosa \& K. Jaegwon (eds), Epistemology. An Anthology, Malden, Blackwell, pp. 58-59, 1963.

M. Gilbert, On Social Facts, London, Routledge,1989.

- Durkheim and social facts, in H. Martins \& W. Pickering, Debating Durkheim, London, Routledge, 1994, pp. 86-109.

- Living Together. Rationality, Sociality and Obligation, Lanham, Rowman \& Littlefields Publishers, 1996.

- Sociality and Responsibility. New Essays, in Plural Subject Theory, Lanhma, Rowman \& Littlefields Publishers, 2000.

A. I. Goldman, Epistemology and Cognition, Cambridge, Harvard University Press, 1986.

- Knowledge in a Social World, Oxford, Oxford University Press, 1999.

- Pathways to Knowledge. Private and Public, Oxford, Oxford University Press, 2002.

W. Heisenberg, Über den anschaulichen Inhalt der quantentheoretischen Kinematic und Mechanik, in «Zeitschrift für Physik», 1927, n 43, pp. 172-198 (cité in Beler, 1999).

P. Jacob, Qu'est-ce qu'une opinion justifiée? Compte rendu de A. Goldman (1986), in P. Jacob (dir.), L'Age de la Science, 2, Epistémologie, Paris, O. Jacob, 1989, pp. 309-332.

T. Kuhn, The Function of Dogma in Scientific Research, in A. C. Crombie (ed.), Scientific Change, New York, Basic, 1963, pp. 347-369 et pp. 386-385.

L. H. Nelson, Who Knows: From Quine to a Feminist Empiricism, Philadelphia, Temple University Press, 1990.

V. Pareto, Traité de sociologie générale, Genève, Droz, 1968 (1 éd. orig. 1916).

W.V. Quine, L'épistémologie devenue naturelle, in W.V. Quine, Relativité de l'ontologie et autres essais, Paris, Aubier Montaigne, 1977 (éd. originale en anglais 1968), chap. 3, pp. 83-105. 
F. Schmitt (ed.), Socializing Epistemology: The Social Dimensions of Knowledge, Lanham, Rowman \& Littlefield, 1994.

F. Schmitt, Social Epistemology, in J. Greco \& E. Sosa (eds), The Blackwell Guide to Epistemology, Malden, Blackwell Publishers, 1999.

J. R. Searle, La construction sociale de la réalité, Paris, Gallimard, 1998.

D. Sperber, Le savoir des anthropologues, Paris, Hermann, 1982.

D. Sperber \& D. Wilson, La pertinence. Communication et cognition, Paris, Minuit, 1989.

D. Terré, Les dérives de l'argumentation scientifique, Paris, PUF, 1998.

P. Veyne, Les Grecs croyaient-ils à leurs mythes?, Paris, Seuil, 1983. 